\title{
Effects of Stressor and Personality Trait on Stress Response
}

\author{
Xiaoyuan Chu ${ }^{1}$, Fuqiang $\mathrm{Fan}^{2}$, Yuan $\mathrm{Li}^{3}$, Jing $\mathrm{Han}^{1} \&$ Ke $\mathrm{Han}^{4}$ \\ ${ }^{1}$ School of Economics and Resource Management, Beijing Normal University, Beijing, China \\ ${ }^{2}$ School of Law, University of International Business and Economics, Beijing, China \\ ${ }^{3}$ Research Department, CIConsulting, Shenzhen, China \\ ${ }^{4}$ Laboratory of Intelligent Communication Navigation and Micro/Nano-Systems, Beijing University of Posts and \\ Telecommunication, Beijing, China
}

Correspondence: Xiaoyuan Chu, School of Economics and Resource Management, Beijing Normal University, Beijing, China. Tel: 86-131-2675-6090. E-mail: seancxy@126.com

Received: May 3, 2015

doi:10.5430/rwe.v6n2p39
Accepted: May 25, 2015

Online Published: June 4, 2015

URL: http://dx.doi.org/10.5430/rwe.v6n2p39

\begin{abstract}
Based on the theoretical analysis, with first-hand data collection and using multiple regression models, this study explored the relationship between personality trait, stressor and stress response. We draw on the following conclusions: (1) Four stressors (work, health, family and social) as well as two personal traits (openness and neuroticism) are predictors of total stress response, wherein neuroticism is a positive predictor while openness a negative predictor; (2) Four stressors (work, health, family and social) and two personal traits (openness and neuroticism) are predictors of physiological stress response, wherein neuroticism is a positive predictor while openness a negative predictor; (3) Four stressors (work, health, family and social) and three personal traits (neuroticism, openness and agreeableness) are predictors of psychological stress response, wherein neuroticism is a positive predictor while openness and agreeableness are negative predictors; (4)Three stressors (health, family and social) and the three personal traits (conscientiousness, neuroticism and openness) are predictors of behavioral stress response, wherein neuroticism is a positive predictor while conscientiousness and openness are negative predictors.
\end{abstract}

Keywords: personality trait, stressor, stress response

Stress is everywhere in modern life. Increasingly more problems are emerging because of stress. Many studies have found the impact of stress on health and well-being (Krishnakumar et al, 2015; Feng et al, 2014; Velden, 2014). And occupational stress is associated with numerous health problems that cost organizations considerable resources (Cardon et al, 2015). Moreover, socio-economic stress not only affects the individuals themselves, but also has a profound impact on their children (Han \& Xiaoyuan Chu, 2014). Similarly, many studies found that personality has a close relationship with reactivity to stress which is closely related to health and some personality traits have a function of "facilitator" to mental health (Cui \& Wang, 2007). For example, toughness is a protective factor for health from stress (Kobasa, 1982). The "Big Five" are relatively stable personalities and some evidence suggests that all five dimensions have a biological-heritable basis (Costa \& McCrae, 1992), suggesting a link to the physiological process underlying stress-related illness and disease (Grant \& Langan, 2007). For instance, some research found that neuroticism has a significant positive correlation with tiredness, extraversion is significantly negatively correlated with tiredness, and agreeableness is negatively correlated with emotional exhaustion and depersonalization, while conscientiousness and emotional exhaustion are negatively correlated (Mills \& Huebner, 1998; Ahart, 2004). However, the mechanism of the effects of stressor and personality trait on stress response is still unknown.

On the basis of previous studies, this study attempts to use primary and secondary school teachers as subjects, investigating the relationship among the stressor, personality traits and stress response, trying to discover related mechanisms.

In order to reveal the relationship between stress response, stressors and personality traits, this paper constructed a basic model as follows:

$$
\text { Re } \text { sponse }_{i}=\beta_{0}+\sum \lambda_{1 j} \text { Stressor }_{j i}+\sum \lambda_{2 k} \text { Personality }_{k i}+\varepsilon_{i}
$$

In the formula, i represents the subjects, $\mathrm{j}$ represents the type of stressors, $\mathrm{k}$ represents the type of personality traits, Response represents the stress response (including four models: the total stress response, physiological stress 
response, psychological stress response and behavioral stress response), Stressor represents pressure source (including work stress, health stress, family stress and social stress), Personality represents personality traits (including extraversion, neuroticism, openness, agreeableness and conscientiousness), and $\varepsilon_{\mathrm{i}}$ is the error term.

\section{Research Methods}

\subsection{Subjects}

460 primary and secondary school teachers were recruited as subjects and 432 questionnaires were returned. After excluding invalid questionnaires, we finally obtained 428 valid questionnaires. The valid response rate is $93.04 \%$. The basic information of the sample is in Table 1 as below.

\subsection{Research Instrument}

\subsubsection{Personality}

Big Five Personality Inventory, namely NEO-Personality Inventory is used to measure the personality traits. This scale is based on the Big Five personality theory and was compiled by the American psychologist Costa Costa and McCrae McRae in 1987. After many years of use and revise, this scale has been of high reliability and validity. As the most authoritative and comprehensive personality test scale, its reliability and validity have been widely recognized. The scale consists of five dimensions, with 25 items included. The five dimensions are extraversion, neuroticism, openness, agreeableness and conscientiousness. The scale uses five scoring system, whose range of scores is 1 to 5 points. Scoring higher or lower indicates some more obvious characteristics in certain aspects.

\subsubsection{Stressor and Stress Response}

Work Stress Scale for Primary and Secondary School Teacher was used to measure stressor and stress response in this study. The scale consists of two parts. The first part is the source of stress, including a total of 36 items in four dimensions. The four dimensions are: work stress, health stress, family stress and social stress. The second part is the stress response, including a total of 17 items in three dimensions. The three dimensions are physiological stress response, psychological stress response and behavioral stress response. It has been testified that the liability and validity of the scale are good. Specifically, the scale uses five scoring system, whose range of scores is 0 to 4 points. Scoring higher or lower indicates some more obvious characteristics in certain aspects.

\subsection{Research Process}

The questionnaires were administrated with the unified instructions. And the questionnaires, with no time limitation, were collected on the spot and checked one by one with invalid ones eliminated. This research employed SPSS19.0 for statistical analysis, which includes analysis of variance, correlation analysis and analysis of regression.

\section{Results}

\subsection{Stress Response and Demographic Variables}

\subsubsection{Stress Response and Marital Status}

The $\mathrm{F}$ test for the total stress response and its dimensions for the married, unmarried and divorced showed that there is a significant difference in the total stress response of subjects with different marital status ( $\mathrm{F}=8.104$, $\mathrm{P}=0.000<0.01)$. So does that in psychological stress response and behavioral stress response $(\mathrm{P}<0.05)$. Through multiple comparisons, the study further found that the differences in total stress response among the married, unmarried and divorced were significant or nearly significant. The divorced had the strongest response, the response of married subjects was in the middle, and the response of unmarried subjects was relatively slight. As with the psychological stress response, the differences between the unmarried subjects and the married and divorced were both significant, but no significant difference was found between the married and the divorced. As with the behavioral response, the difference between the unmarried and married was significant (The result is shown in Table 1).

\subsubsection{Stress Response and Type of School}

The $\mathrm{F}$ test for the total stress response and its dimensions of elementary, junior and senior high school teachers showed that there is a significant difference in the total stress response of subjects teaching in different types of schools $(\mathrm{F}=5.180, \mathrm{P}=0.006<0.01)$. So does that in physiological stress response and psychological stress response $(\mathrm{P}<0.05)$. Through multiple comparisons, the study further found that the differences in total stress response, physiological stress response and psychological stress response between the individuals of elementary schools and that of the high school were significant, but no significant difference was found between junior high school teachers and senior high school teachers in all the dimensions mentioned above. The elementary school teachers showed more pronounced stress response (The result is shown in Table 1). 


\subsubsection{Stress Response and Service Year}

The $\mathrm{F}$ test for the total stress response and its dimensions for individuals of different service years showed that there is a significant difference in the total stress response of subjects with different service years $(\mathrm{F}=3.536, \mathrm{P}=0.015<0.05)$. So does that in the three dimensions of stress response $(\mathrm{P}<0.05)$. Through multiple comparisons, the study further found that the differences in total stress response, psychological stress response and behavioral stress response between the subjects having 0-10 years of service and the subjects having 10-20 years of service were significant. And the differences in physiological stress response between the subjects having 0-10 years of service and the subjects having s over 20 years of service were significant. It can be concluded that there is a close relationship between the subjects' stress response and their service years. Compared to the novice subjects, subjects having rich teaching experience are more likely to face greater stress, and the subjects having 10-20 of seniority may face the greatest stress. Subjects having 20 years of service mainly suffer from physiological stress (The result is shown in Table 1).

\subsubsection{Stress Response and Fertilities}

The $\mathrm{T}$ test for the total stress response and its dimensions for different fertilities showed that there is a significant difference in the total stress response of subjects who have child(ren) and who don't ( $F=7.664, \mathrm{P}=0.006<0.01)$. So does that in behavioral stress response $(\mathrm{P}<0.05)$. Subjects who have child(ren) get higher total stress response and behavioral stress response, but their differences in physiological and psychological stress response are statistically significant (The result is shown in Table 1).

\subsubsection{Stress Response and Gender}

The $\mathrm{T}$ test for the total stress response and its dimensions for different gender showed that there is a significant difference in the behavioral stress response between male and female subjects $(\mathrm{P}<0.05)$. Female subjects have higher behavioral stress response than the male subjects. But there was no significant difference in total stress response, physiological stress response and psychological stress response (The result is shown in Table 1).

Table 1. Basic information of the sample and the F-test for the stress response

\begin{tabular}{|c|c|c|c|c|c|c|c|c|}
\hline $\begin{array}{c}\text { Demographic } \\
\text { Variable }\end{array}$ & & $\mathrm{N}$ & Percentage & $\begin{array}{l}\text { Statistical } \\
\text { value }\end{array}$ & $\begin{array}{l}\text { Physiological } \\
\text { Stress Response }\end{array}$ & $\begin{array}{l}\text { Psychological } \\
\text { Stress Response }\end{array}$ & $\begin{array}{c}\text { Behavioral } \\
\text { Stress } \\
\text { Response }\end{array}$ & $\begin{array}{c}\text { Total Stress } \\
\text { Response }\end{array}$ \\
\hline \multirow{3}{*}{$\begin{array}{l}\text { Marital } \\
\text { status }\end{array}$} & Unmarried & 85 & 20.4 & & $8.22 \pm 3.704$ & $11.81 \pm 6.089$ & $2.67 \pm 1.572$ & $21.91 \pm 9.446$ \\
\hline & Married & 323 & 77.5 & & $8.77 \pm 3.777$ & $14.6 \pm 6.762$ & $4.17 \pm 2.517$ & $27.4 \pm 11.295$ \\
\hline & Divorced & 9 & 2.2 & & $10.25 \pm 3.732$ & $18.38 \pm 5.605$ & $4.6 \pm 2.608$ & $38.25 \pm 12.997$ \\
\hline \multirow{7}{*}{$\begin{array}{l}\text { Types of } \\
\text { School }\end{array}$} & & & & $\mathrm{F}$ & 1.41 & 7.636 & 9.685 & 8.104 \\
\hline & & & & $\mathrm{P}$ & 0.245 & 0.001 & 0.000 & 0.000 \\
\hline & Elementary & 154 & 38.1 & & $10.16 \pm 3.733$ & $15.25 \pm 7.170$ & $4.23 \pm 2.519$ & $29.24 \pm 12.596$ \\
\hline & Junior High & 77 & 19.1 & & $7.89 \pm 3.486$ & $13.29 \pm 6.823$ & $3.35 \pm 2.077$ & $25.19 \pm 10.487$ \\
\hline & Senior High & 173 & 42.8 & & $7.66 \pm 3.423$ & $13.33 \pm 6.283$ & $3.89 \pm 2.508$ & $24.92 \pm 10.251$ \\
\hline & & & & $\mathrm{F}$ & 22.082 & 3.863 & 2.87 & 5.18 \\
\hline & & & & $\mathrm{P}$ & 0.000 & 0.022 & 0.058 & 0.006 \\
\hline \multirow{6}{*}{ Service Year } & $\leq 5$ & 71 & 17 & & $8.07 \pm 3.969$ & $12.32 \pm 5.947$ & $2.96 \pm 2.345$ & $23.11 \pm 10.484$ \\
\hline & $5<\leq 10$ & 146 & 35 & & $8.21 \pm 3.472$ & $13.81 \pm 6.470$ & $3.65 \pm 2.182$ & $25.62 \pm 10.093$ \\
\hline & $10<\leq 20$ & 144 & 34.5 & & $9.01 \pm 4.022$ & $15.44 \pm 7.266$ & $4.59 \pm 2.443$ & $28.93 \pm 12.41$ \\
\hline & $>20$ & 56 & 13.4 & & $9.88 \pm 3.390$ & $13.87 \pm 6.885$ & $3.85 \pm 2.912$ & $26.32 \pm 11.267$ \\
\hline & & & & $\mathrm{F}$ & 3.636 & 3.643 & 6.246 & 3.536 \\
\hline & & & & $\mathrm{P}$ & 0.013 & 0.013 & 0.000 & 0.015 \\
\hline \multirow{4}{*}{ Child(ren) } & With & 236 & 66.5 & & $8.86 \pm 3.81$ & $14.55 \pm 6.911$ & $4.44 \pm 2.684$ & $27.73 \pm 11.958$ \\
\hline & Without & 119 & 33.5 & & $8.14 \pm 3.379$ & $13.15 \pm 6.085$ & $2.90 \pm 1.429$ & $24.14 \pm 9.408$ \\
\hline & & & & $\mathrm{F}$ & 3.063 & 3.405 & 33.587 & 7.664 \\
\hline & & & & $\mathrm{P}$ & 0.081 & 0.066 & 0.000 & 0.006 \\
\hline \multirow{4}{*}{ Gender } & Male & 97 & 23.4 & & $8.19 \pm 3.756$ & $13.69 \pm 6.629$ & $4.40 \pm 3.007$ & $26.24 \pm 10.444$ \\
\hline & Female & 317 & 76.6 & & $8.81 \pm 3.78$ & $14.22 \pm 6.844$ & $3.77 \pm 2.232$ & $26.63 \pm 11.562$ \\
\hline & & & & $\mathrm{F}$ & 2.01 & 0.427 & 4.093 & 0.074 \\
\hline & & & & $\mathrm{P}$ & 0.157 & 0.514 & 0.044 & 0.786 \\
\hline
\end{tabular}




\subsection{Correlation Analysis of Stressors and Stress Response}

A correlation analysis of the stressor (as well as its dimensions) and the total stress response (as well as its dimensions) was conducted. As shown in Table 2, all the dimensions of stressors are significantly positively correlated with stress response and its dimensions.

Table 2. Correlation matrix of variables

\begin{tabular}{|c|c|c|c|c|c|c|c|c|c|c|c|c|c|}
\hline Variable & 1 & 2 & 3 & 4 & 5 & 6 & 7 & 8 & 9 & 10 & 11 & 12 & 13 \\
\hline 1 Neuroticism & 1 & & & & & & & & & & & & \\
\hline 2 Extraversion & .028 & 1 & & & & & & & & & & & \\
\hline 3 Openness & $.114 *$ & $.203 * *$ & 1 & & & & & & & & & & \\
\hline 4 Agreeableness & $-.155^{* *}$ & $.494 * *$ & .035 & 1 & & & & & & & & & \\
\hline 5 Conscientiousness & $-.183 * *$ & $.164 * *$ & -.070 & $.301^{* *}$ & 1 & & & & & & & & \\
\hline 6 Stressor (work) & $.275 * *$ & $-.213 * *$ & $-.160 * *$ & $-.223 * *$ & $-.109 *$ & 1 & & & & & & & \\
\hline 7 Stressor (health) & $.340 * *$ & -.061 & -.078 & $-.193 * *$ & $-.160 * *$ & $.643^{* *}$ & 1 & & & & & & \\
\hline 8 Stressor (family) & .055 & $-.182 * *$ & $-.100 *$ & -.087 & -.017 & $.457^{* *}$ & $.285^{* *}$ & 1 & & & & & \\
\hline 9 Stressor (social) & $.127 * *$ & $-.198 * *$ & $-.168 * *$ & $-.153 * *$ & -.080 & $.591 * *$ & $.337 * *$ & $.418^{* *}$ & 1 & & & & \\
\hline 10 Physiological & $.247 * *$ & $-.115^{*}$ & $-.182 * *$ & -.088 & -.082 & $.476^{* *}$ & $.447^{* *}$ & $.352 * *$ & $.382 * *$ & 1 & & & \\
\hline \multicolumn{14}{|l|}{ Stress Response } \\
\hline 11 Psychological & $.327 * *$ & $-.157 * *$ & $-.203 * *$ & $-.237 * *$ & $-.163 * *$ & $.579 * *$ & $.536^{* *}$ & $.357 * *$ & $.460 * *$ & $.665^{* *}$ & 1 & & \\
\hline \multicolumn{14}{|l|}{ Stress Response } \\
\hline 12 Behavioral Stress & $.249^{* *}$ & -.077 & $-.138 * *$ & $-.162 * *$ & $-.175^{* *}$ & $.415^{* *}$ & $.483^{* *}$ & $.315^{* *}$ & $.347 * *$ & $.539^{* *}$ & $.673^{* *}$ & 1 & \\
\hline \multicolumn{14}{|l|}{ Response } \\
\hline 13 Total Stress & $.318^{* *}$ & $-.135^{* *}$ & $-.202 * *$ & $-.191 * *$ & $-.163^{* *}$ & $.568 * *$ & $.565^{* *}$ & $.393 * *$ & $.460 * *$ & $.841 * *$ & $.908^{* *}$ & $.850 * *$ & 1 \\
\hline \multicolumn{14}{|l|}{ Response } \\
\hline Minimum & 20 & 33 & 25 & 20 & 20 & .05 & 0 & 0 & 0 & 0 & 0 & 0 & .20 \\
\hline Maximum & 80 & 72 & 67 & 71 & 69 & 3.48 & 4.00 & 4.00 & 4.00 & 4.00 & 3.88 & 3.75 & 10.96 \\
\hline Mean & 48.62 & 50.72 & 48.92 & 55.71 & 55.00 & 1.83 & 1.70 & 1.67 & 2.13 & 1.73 & 1.78 & 1.30 & 4.82 \\
\hline
\end{tabular}

Note: $* *$ and $*$ indicate that the coefficient is significant at the 0.01 and 0.05 levels respectively.

\subsection{Correlation Analysis of Personality Trait and Stress Response}

The correlation analysis of personality trait and stress response was conducted. The results are shown in Table 2 and we can see that the five dimensions of personality trait have different effects on the total stress response and its dimensions:

(1) Neuroticism is positively correlated with the total stress response and all its dimensions;

(2) Extraversion is negatively correlated with the total stress response, physiological stress response, psychological stress response, but not significantly correlated with behavioral stress response;

(3) Openness is negatively correlated with total stress response and all its dimensions;

(4) Agreeableness is negatively correlated with total stress response, psychological stress response and behavioral stress response, but not significantly correlated with physiological stress response;

(5) Conscientiousness is negatively correlated with total stress response, psychological stress response and behavioral stress response, but not significantly correlated with physiological stress response.

Overall, the correlations between dimensions of personality trait and total stress response are statistically significant, indicating individuals with different personalities have significantly different stress response.

\subsection{Stepwise Regression Analysis of Personalities on Stress Response}

To better understand the impact of personality traits on the stress response, we conducted a regression analysis, with total stress response, physiological stress response, psychological stress response and behavioral stress response as predicted variable respectively, and personality traits as predictive variable. The study selected stepwise regression as the method. First, it separately calculated for each predictive variable's contribution on the predicted variable, selecting the variable having the largest contribution into the equation. Then it recalculated the remaining predictive variable's contribution to the predicted variable respectively, and examined whether the introduction of a new 
variable had made the equation no longer statistically significant. If so, the new variable had to be removed and the contributions of all the predictive variables to the predicted variables had to be recalculated. This process was conducted until no variables could be removed from and no variables could be introduced into the equation. The regression results are shown in Table 3.

Table 3. Stepwise regression analysis of personality on dimensions of stress response

\begin{tabular}{lccccc}
\hline Predicted variable & Predictive variable & Standardized $\beta$ & $\mathrm{T}$ & $\mathrm{P}$ & Adjusted R $^{2}$ \\
\hline Physiological Stress Response & Neuroticism & 0.271 & 5.864 & 0.000 & 0.101 \\
& Openness & -0.213 & -4.606 & 0.000 & \\
Psychological Stress Response & Neuroticism & 0.326 & 7.350 & 0.000 & 0.191 \\
& Openness & -0.234 & -5.334 & 0.000 & \\
& Agreeableness & -0.178 & -4.030 & 0.000 & \\
Behavioral Stress Response & Neuroticism & 0.242 & 5.173 & 0.000 & 0.103 \\
& Openness & -0.175 & -3.794 & 0.000 & \\
Total Stress Response & Conscientiousness & -0.142 & -3.053 & 0.002 & \\
& Neuroticism & 0.324 & 7.214 & 0.000 & 0.170 \\
& Openness & -0.234 & -5.267 & 0.000 & \\
& Agreeableness & -0.132 & -2.961 & 0.003 & \\
\hline
\end{tabular}

As shown in Table 3 shows that:

Neuroticism and openness entered into the regression equation on physiological stress response, with a predictive power of $10.1 \%$;

Neuroticism, openness and agreeableness entered into the regression equation on psychological stress response, with a predictive power of $19.1 \%$;

Neuroticism, openness and conscientiousness entered into the regression equation on behavioral stress response, with a predictive power of $10.3 \%$;

Neuroticism, openness and agreeableness entered into the regression equation on total stress response, with a predictive power of $17.0 \%$.

\subsection{Forced Entry Regression Analysis of Stressors and Personality to Stress Response}

In order to further reveal the combined effect of stressor and personality trait on the stress response, we took total stress response, physiological stress response, psychological stress response and behavioral stress response respectively as the predicted variable, and work stress, health stress, family stress, social stress and personality traits as the predictive variables to do the regression analysis. The results are shown in Tables 4-7.

As shown in Table 4, in consideration of the stressor, the neuroticism, openness and conscientiousness are all significant in the regression model of the total stress response. The model 7 indicates that neuroticism, openness, health stress, family stress and social stress enter into the regression equation to total stress response, with the predictive power of $48.3 \%$.

As shown in Table 5, in consideration of the stressor, the openness is significant in the regression model of the physiological stress response. The model 7 indicates that neuroticism, openness, health stress, family stress and social stress enter into the regression equation to physiological stress response, with the predictive power of $35.5 \%$.

As shown in Table 6, in consideration of the stressor, the neuroticism is significant in the regression model of the psychological stress response. The model 7 indicates that neuroticism, openness, health stress, work stress and social stress enter into the regression equation to psychological stress response, with the predictive power of $45.6 \%$.

As shown in Table 7, in consideration of the stressor, the conscientiousness is significant in the regression model of the behavioral stress response. The model 7 indicates that the openness, conscientiousness, health stress, family stress and social stress enter into the regression equation to behavioral stress response, with the predictive power of $31.2 \%$. 
Table 4. Personality, stressor and total stress response (Predicted variable: total stress response)

\begin{tabular}{|c|c|c|c|c|c|c|c|}
\hline Predictive variable & Model 1 & Model 2 & Model 3 & Model 4 & Model 5 & Model 6 & Model 7 \\
\hline \multicolumn{8}{|l|}{ Personality } \\
\hline Neuroticism & & $\begin{array}{l}.118 * * \\
(2.97)\end{array}$ & & & & & $\begin{array}{l}.123 * * \\
(3.050)\end{array}$ \\
\hline Extraversion & & & $\begin{array}{l}-.008 \\
(-.211)\end{array}$ & & & & $\begin{array}{l}.020 \\
(.448)\end{array}$ \\
\hline Openness & & & & $\begin{array}{l}-.082^{*} \\
(-2.169)\end{array}$ & & & $\begin{array}{l}-.108^{* *} \\
(-2.828)\end{array}$ \\
\hline Agreeableness & & & & & $\begin{array}{l}-.032 \\
(-.839)\end{array}$ & & $\begin{array}{l}-.015 \\
(-.349)\end{array}$ \\
\hline Conscientiousness & & & & & & $\begin{array}{l}-.076^{*} \\
(-2.052)\end{array}$ & $\begin{array}{l}-.071 \\
(-1.847)\end{array}$ \\
\hline \multicolumn{8}{|l|}{ Stressor } \\
\hline Stressor (work) & $\begin{array}{l}.138^{*} \\
(2.448)\end{array}$ & $\begin{array}{l}.118^{*} \\
(2.096)\end{array}$ & $\begin{array}{l}.137^{*} \\
(2.389)\end{array}$ & $\begin{array}{l}.133^{*} \\
(2.363)\end{array}$ & $\begin{array}{l}.133^{*} \\
(2.341)\end{array}$ & $\begin{array}{l}.136^{*} \\
(2.416)\end{array}$ & $\begin{array}{l}.110 \\
(1.940)\end{array}$ \\
\hline Stressor (health) & $\begin{array}{l}.365^{* * *} \\
(7.675)\end{array}$ & $\begin{array}{l}.332 * * * \\
(6.836)\end{array}$ & $\begin{array}{l}.367 * * * \\
(7.637)\end{array}$ & $\begin{array}{l}.369 * * * \\
(7.779)\end{array}$ & $\begin{array}{l}.363 * * * \\
(7.607)\end{array}$ & $\begin{array}{l}.355^{* * *} \\
(7.435)\end{array}$ & $\begin{array}{l}.321 * * * \\
(6.582)\end{array}$ \\
\hline Stressor (family) & $\begin{array}{l}.107^{*} \\
(2.510)\end{array}$ & $\begin{array}{l}.126^{* *} \\
(2.948)\end{array}$ & $\begin{array}{l}.106^{*} \\
(2.484)\end{array}$ & $\begin{array}{l}.107^{*} \\
(2.521)\end{array}$ & $\begin{array}{l}.109^{*} \\
(2.550)\end{array}$ & $\begin{array}{l}.110^{*} \\
(2.602)\end{array}$ & $\begin{array}{l}.132 * * \\
(3.111)\end{array}$ \\
\hline Stressor (social) & $\begin{array}{l}.217 * * * \\
(4.671)\end{array}$ & $\begin{array}{l}.217 * * * \\
(4.718)\end{array}$ & $\begin{array}{l}.216^{* * *} \\
(4.633)\end{array}$ & $\begin{array}{l}.205^{* * *} \\
(4.421)\end{array}$ & $\begin{array}{l}.214^{* * *} \\
(4.605)\end{array}$ & $\begin{array}{l}.213 * * * \\
(4.613)\end{array}$ & $\begin{array}{l}.199 * * * \\
(4.336)\end{array}$ \\
\hline \multicolumn{8}{|l|}{ Control variable } \\
\hline Grade & $\begin{array}{l}-.131 * * * \\
(-3.528)\end{array}$ & $\begin{array}{l}-.125^{* *} \\
(-3.398)\end{array}$ & $\begin{array}{l}-.132 * * * \\
(-3.530)\end{array}$ & $\begin{array}{l}-.133 * * * \\
(-3.587)\end{array}$ & $\begin{array}{l}-.134 * * * \\
(-3.582)\end{array}$ & $\begin{array}{l}-.135 * * * \\
(-3.624)\end{array}$ & $\begin{array}{l}-.130 * * * \\
(-3.546)\end{array}$ \\
\hline Marital status & $\begin{array}{l}.152^{* * *} \\
(4.065)\end{array}$ & $\begin{array}{l}.144 * * * \\
(3.869)\end{array}$ & $\begin{array}{l}.152 * * * \\
(4.026)\end{array}$ & $\begin{array}{l}.137 * * * \\
(3.616)\end{array}$ & $\begin{array}{l}.150^{* * *} \\
(3.987)\end{array}$ & $\begin{array}{l}.151^{* * *} \\
(4.046)\end{array}$ & $\begin{array}{l}.123 * * \\
(3.258)\end{array}$ \\
\hline$R^{2}$ & .471 & .482 & .471 & .477 & .472 & .476 & .497 \\
\hline Adjusted $R^{2}$ & .463 & .473 & .461 & .468 & .462 & .467 & .483 \\
\hline$N$ & 399 & 399 & 399 & 399 & 399 & 399 & 399 \\
\hline
\end{tabular}

Note: ***,** and * indicate that the coefficient is significant at the $0.01,0.05$ and 0.1 levels, respectively the same below.

Table 5. Personality, stressor and physiological stress response (Predicted variable: physiological stress response)

\begin{tabular}{|c|c|c|c|c|c|c|c|}
\hline Predictive variable & Model 1 & Model 2 & Model 3 & Model 4 & Model 5 & Model 6 & Model 7 \\
\hline \multicolumn{8}{|l|}{ Personality } \\
\hline Neuroticism & & $\begin{array}{l}.085 \\
(1.919)\end{array}$ & & & & & $\begin{array}{l}.102^{*} \\
(2.282)\end{array}$ \\
\hline Extraversion & & & $\begin{array}{l}-.029 \\
(-.685)\end{array}$ & & & & $\begin{array}{l}-.045 \\
(-.918)\end{array}$ \\
\hline Openness & & & & $\begin{array}{l}-.084^{*} \\
(-2.028)\end{array}$ & & & $\begin{array}{l}-.093^{*} \\
(-2.174)\end{array}$ \\
\hline Agreeableness & & & & & $\begin{array}{l}.027 \\
(.635)\end{array}$ & & $\begin{array}{l}.062 \\
(1.272)\end{array}$ \\
\hline Conscientiousness & & & & & & $\begin{array}{l}-.027 \\
(-.657)\end{array}$ & $\begin{array}{l}-.034 \\
(-.793)\end{array}$ \\
\hline
\end{tabular}




\begin{tabular}{llllllll}
\hline Stressor & & & & & & & \\
Stressor (work) & .112 & .097 & .106 & .106 & .116 & .111 & .088 \\
& $(1.792)$ & $(1.554)$ & $(1.675)$ & $(1.710)$ & $(1.849)$ & $(1.777)$ & $(1.389)$ \\
Stressor (health) & $.270^{* * *}$ & $.245^{* * *}$ & $.274^{* * *}$ & $.273^{* * *}$ & $.272^{* * *}$ & $.266^{* * *}$ & $.251^{* * *}$ \\
& $(5.130)$ & $(4.552)$ & $(5.172)$ & $(5.215)$ & $(5.156)$ & $(5.024)$ & $(4.611)$ \\
Stressor (family) & $.130^{* *}$ & $.143^{* *}$ & $.127^{* *}$ & $.130^{* *}$ & $.128^{* *}$ & $.131^{* *}$ & $.140^{* *}$ \\
& $(2.761)$ & $(3.025)$ & $(2.700)$ & $(2.771)$ & $(2.720)$ & $(2.783)$ & $(2.954)$ \\
Stressor (social) & $.149^{* *}$ & $.149^{* *}$ & $.146^{* *}$ & $.137^{* *}$ & $.151^{* *}$ & $.148^{* *}$ & $.135^{* *}$ \\
& $(2.909)$ & $(2.919)$ & $(2.842)$ & $(2.676)$ & $(2.942)$ & $(2.881)$ & $(2.635)$ \\
Control variable & & & & & & & \\
Grade & $-.261^{* * *}$ & $-.257^{* * *}$ & $-.263^{* * *}$ & $-.263^{* * *}$ & $-.259^{* * *}$ & $-.262^{* * *}$ & $-.257^{* * *}$ \\
& $(-6.346)$ & $(-6.255)$ & $(-6.371)$ & $(-6.410)$ & $(-6.271)$ & $(-6.363)$ & $(-6.261)$ \\
Marital status & $.084^{*}$ & .078 & $.082^{*}$ & .069 & $.086^{*}$ & $.084^{*}$ & .060 \\
& $(2.038)$ & $(1.894)$ & $(1.969)$ & $(1.639)$ & $(2.079)$ & $(2.025)$ & $(1.429)$ \\
$R^{2}$ & .355 & .361 & .356 & .362 & .356 & .356 & .373 \\
Adjusted $R^{2}$ & .345 & .350 & .344 & .350 & .344 & .344 & .355 \\
$N$ & 399 & 399 & 399 & 399 & 399 & 399 & 399 \\
\hline
\end{tabular}

Table 6. Personality, stressor and psychological stress response (Predicted variable: psychological stress response)

\begin{tabular}{llllllll}
\hline Predictive variable & Model 1 & Model 2 & Model 3 & Model 4 & Model 5 & Model 6 & Model 7 \\
\hline Personality & & & & & & & \\
Neuroticism & & $.137^{* *}$ & & & & & $.140^{* *}$ \\
& & $(3.366)$ & & & & & $(3.381)$ \\
Extraversion & & & -.023 & & & & .021 \\
& & & $(-.588)$ & & & & $(.464)$ \\
Openness & & & & -.072 & & & $-.101^{*}$ \\
& & & & $(-1.868)$ & & & $(-2.562)$ \\
Agreeableness & & & & & -.073 & & -.061 \\
& & & & & $(-1.872)$ & & $(-1.349)$ \\
Conscientiousness & & & & & & -.073 & -.053 \\
& & & & & & $(-1.909)$ & $(-1.353)$ \\
Stressor & & & & & & & \\
Stressor (work) & $.220^{* * *}$ & $.197^{* *}$ & $.215^{* * *}$ & $.216^{* * *}$ & $.208^{* * *}$ & $.218^{* * *}$ & $.183^{* *}$ \\
& $(3.787)$ & $(3.403)$ & $(3.661)$ & $(3.715)$ & $(3.574)$ & $(3.761)$ & $(3.146)$ \\
Stressor (health) & $.295^{* * *}$ & $.255^{* * *}$ & $.298^{* * *}$ & $.298^{* * *}$ & $.289^{* * *}$ & $.284^{* * *}$ & $.243^{* * *}$ \\
& $(6.017)$ & $(5.133)$ & $(6.039)$ & $(6.094)$ & $(5.910)$ & $(5.791)$ & $(4.873)$ \\
Stressor (family) & .048 & .070 & .046 & .048 & .053 & .052 & .078 \\
& $(1.098)$ & $(1.601)$ & $(1.050)$ & $(1.101)$ & $(1.202)$ & $(1.179)$ & $(1.794)$ \\
Stressor (social) & $.230^{* * *}$ & $.230^{* * *}$ & $.228^{* * *}$ & $.220^{* * *}$ & $.151^{* *}$ & $.227^{* * *}$ & $.211^{* * *}$ \\
& $(4.827)$ & $(4.891)$ & $(4.760)$ & $(4.604)$ & $(2.942)$ & $(4.772)$ & $(4.481)$ \\
Control variable & & & & & & & \\
Grade & -.056 & -.049 & -.057 & -.057 & -.062 & -.059 & -.057 \\
& $(-1.460)$ & $(-1.295)$ & $(-1.491)$ & $(-1.502)$ & $(-1.608)$ & $(-1.542)$ & $(-1.501)$ \\
Marital status & $.177^{* * *}$ & $.167^{* * *}$ & $.175^{* * *}$ & $.164^{* * *}$ & $.171^{* * *}$ & $.176^{* * *}$ & $.145^{* * *}$ \\
& $(4.593)$ & $(4.386)$ & $(4.521)$ & $(4.186)$ & $(4.450)$ & $(4.575)$ & $(3.742)$ \\
$R^{2}$ & .440 & .456 & .441 & .445 & .445 & .446 & .471 \\
Adjusted $R^{2}$ & .432 & .446 & .431 & .435 & .435 & .436 & .456 \\
$N$ & 399 & 399 & 399 & 399 & 399 & 399 & 399 \\
\hline
\end{tabular}


Table 7. Personality, stressor and behavioral stress response (Predicted variable: behavioral stress response)

\begin{tabular}{|c|c|c|c|c|c|c|c|}
\hline Predictive variable & Model 1 & Model 2 & Model 3 & Model 4 & Model 5 & Model 6 & Model 7 \\
\hline \multicolumn{8}{|l|}{ Personality } \\
\hline \multirow[t]{2}{*}{ Neuroticism } & & .080 & & & & & .073 \\
\hline & & $(1.761)$ & & & & & $(1.577)$ \\
\hline \multirow[t]{2}{*}{ Extraversion } & & & .032 & & & & .072 \\
\hline & & & $(.730)$ & & & & $(1.434)$ \\
\hline \multirow[t]{2}{*}{ Openness } & & & & -.056 & & & $-.087^{*}$ \\
\hline & & & & $(-1.288)$ & & & $(-1.968)$ \\
\hline \multirow[t]{2}{*}{ Agreeableness } & & & & & -.030 & & -.034 \\
\hline & & & & & $(-.694)$ & & $(-.671)$ \\
\hline \multirow[t]{2}{*}{ Conscientiousness } & & & & & & $-.096^{*}$ & $-.097 *$ \\
\hline & & & & & & $(-2.260)$ & $(-2.186)$ \\
\hline \multicolumn{8}{|l|}{ Stressor } \\
\hline \multirow[t]{2}{*}{ Stressor (work) } & .017 & .003 & .024 & .013 & .012 & .014 & .006 \\
\hline & $(.263)$ & $(.049)$ & $(.364)$ & $(.207)$ & $(.186)$ & $(.218)$ & $(.088)$ \\
\hline \multirow[t]{2}{*}{ Stressor (health) } & $.385^{* * *}$ & $.362 * * *$ & $.380^{* * *}$ & $.387^{* * *}$ & $.383 * * *$ & $.372 * * *$ & $.341 * * *$ \\
\hline & $(7.082)$ & $(6.488)$ & $(6.942)$ & (7.127) & $(7.022)$ & $(6.829)$ & $(6.068)$ \\
\hline \multirow[t]{2}{*}{ Stressor (family) } & $.105^{*}$ & $.118^{*}$ & $.108^{*}$ & $.105^{*}$ & $.107 *$ & $.110^{*}$ & $.130 * *$ \\
\hline & $(2.166)$ & $(2.410)$ & $(2.213)$ & $(2.167)$ & $(2.199)$ & $(2.268)$ & (2.644) \\
\hline \multirow[t]{2}{*}{ Stressor (social) } & $.178 * *$ & $.178 * *$ & $.181 * *$ & $.170 * *$ & $.175^{* *}$ & $.173 * *$ & $.166^{* *}$ \\
\hline & $(3.355)$ & (3.364) & $(3.402)$ & (3.193) & $(3.301)$ & (3.289) & $(3.131)$ \\
\hline \multicolumn{8}{|l|}{ Control variable } \\
\hline \multirow[t]{2}{*}{ Grade } & -.035 & -.031 & -.033 & -.036 & -.038 & -.039 & -.036 \\
\hline & $(-.830)$ & $(.462)$ & $(-.785)$ & $(-.856)$ & $(-.882)$ & $(-.926)$ & $(-.844)$ \\
\hline \multirow[t]{2}{*}{ Marital status } & $.128 * *$ & $.122 * *$ & $.131 * *$ & $.118^{* *}$ & $.126^{* *}$ & $.126^{* *}$ & $.109 *$ \\
\hline & (2.991) & $(2.858)$ & (3.042) & $(2.706)$ & $(2.927)$ & (2.968) & $(2.500)$ \\
\hline$R^{2}$ & .309 & .315 & .310 & .312 & .310 & .318 & .331 \\
\hline Adjusted $R^{2}$ & .299 & .303 & .298 & .300 & .298 & .306 & .312 \\
\hline$N$ & 399 & 399 & 399 & 399 & 399 & 399 & 399 \\
\hline
\end{tabular}

2.6 Stepwise Regression Analysis of Personality and Stressors on Stress Response

To further reveal the predictive effect of personality trait and stressor on the stress response, we conducted a stepwise regression analysis, with physiological stress response, psychological stress response, behavioral stress response and total stress response as predicted variable respectively, and neuroticism, extraversion, openness, agreeableness, conscientiousness, stressor (work), stressor (health), stressor (family) and stressor (social) as predictive variables. The regression results are shown in Table 8. 
Table 8. Stepwise regression analysis of personality and stressors on dimensions of stress response

\begin{tabular}{|c|c|c|c|c|c|}
\hline Predicted variable & Predictive variable & Standardized $\beta$ & $\mathrm{T}$ & $\mathrm{P}$ & Adjusted $\mathrm{R}^{2}$ \\
\hline \multirow{6}{*}{$\begin{array}{l}\text { Physiological } \\
\text { Stress Response }\end{array}$} & Stressor (work) & .132 & 2.068 & .039 & \multirow[t]{6}{*}{0.309} \\
\hline & Stressor (health) & .223 & 4.134 & .000 & \\
\hline & Stressor (family) & .156 & 3.355 & .001 & \\
\hline & Stressor (social) & .128 & 2.506 & .013 & \\
\hline & Openness & -.120 & -2.903 & .004 & \\
\hline & Neuroticism & .123 & 2.828 & .005 & \\
\hline \multirow{7}{*}{$\begin{array}{l}\text { Psychological } \\
\text { Stress Response }\end{array}$} & Stressor (work) & .191 & 3.341 & .001 & \multirow[t]{7}{*}{0.446} \\
\hline & Stressor (health) & .246 & 5.083 & .000 & \\
\hline & Stressor (social) & .166 & 3.621 & .000 & \\
\hline & Neuroticism & .167 & 4.253 & .000 & \\
\hline & Openness & -.132 & -3.545 & .000 & \\
\hline & Stressor (family) & .100 & 2.408 & .016 & \\
\hline & Agreeableness & -.082 & -2.202 & .028 & \\
\hline \multirow{6}{*}{$\begin{array}{l}\text { Behavioral Stress } \\
\text { Response }\end{array}$} & Stressor (health) & .339 & 7.303 & .000 & \multirow[t]{6}{*}{0.301} \\
\hline & Stressor (social) & .137 & 2.950 & .003 & \\
\hline & Stressor (family) & .144 & 3.183 & .002 & \\
\hline & Conscientiousness & -.095 & -2.285 & .023 & \\
\hline & Neuroticism & .101 & 2.306 & .022 & \\
\hline & Openness & -.092 & -2.214 & .027 & \\
\hline \multirow{6}{*}{$\begin{array}{l}\text { Total Stress } \\
\text { Response }\end{array}$} & Stressor (work) & .129 & 2.285 & .023 & \multirow[t]{6}{*}{0.460} \\
\hline & Stressor (health) & .317 & 6.653 & .000 & \\
\hline & Stressor (social) & .171 & 3.783 & .000 & \\
\hline & Stressor (family) & .151 & 3.674 & .000 & \\
\hline & Neuroticism & .160 & 4.147 & .000 & \\
\hline & Openness & -.131 & -3.573 & .000 & \\
\hline
\end{tabular}

The data in Table 8 indicates that:

Stressor (work), stressor (health), stressor (family) and stressor (social), openness and neuroticism entered into the regression equation on physiological stress response, with a predictive power of 30.9\%;

Stressor (work), stressor (health), stressor (family) and stressor (social), neuroticism, openness and agreeableness entered into the regression equation on psychological stress response, with a predictive power of $44.6 \%$;

Stressor (health), stressor (family) and stressor (social), conscientiousness, neuroticism and openness entered into the regression equation on behavioral stress response, with a predictive power of $30.1 \%$;

Stressor (work), stressor (health), stressor (social) and stressor (family), neuroticism and openness entered into the regression equation on total stress response, with a predictive power of $46.0 \%$.

This result generally agrees with the above-mentioned results of the forced entry regression model.

\section{Discussion}

\subsection{Effect of Personality Trait on Stress Response}

Neuroticism reflects the individual's emotional adjustment process, mainly the tendency of individual to experience negative emotions and emotional instability. Individuals high in neuroticism exhibit obvious tendency of annoyance, insecurity and self-pity. They tend to be anxious, hostile, depressed, impulsive and fragile. The study found that neuroticism is significantly positively correlated with stress response. Individuals of high neuroticism tend to respond more intensely to external stressful stimulation than other people. They have relatively poor ability to adjust and cope with their own emotional states, which usually leads to bad mood. Their abilities of thinking, 
decision-making and dealing with stressor are relatively poor as well. So they are more likely to feel danger and threat, and easily to get nervous, feared, worried and anxious. These emotional responses can be reflected in the individuals' physiological, psychological and behavioral aspects.

Openness includes traits of imagination, aesthetic appreciation, rich emotions, seeking of difference, creativity and intelligence. Individuals high in openness are imaginative. They would seek for changes and are more automatic. They are curious and free people who have broad interests and pursue novelty. We found that openness had significantly negative correlation with total stress response, physiological stress response, psychological stress response and behavioral stress response in this study. This may attributes to the high tolerance for strange situation of individuals high in openness. Because their comfort zones are broader, they will feel relatively less suffering when facing different stressful situations.

Agreeableness includes traits of trust, altruism, frankness, modesty and empathy. Individuals of high agreeableness are more enthusiastic, trusting and helpful. They are optimistic about human nature, believing in innate purity. The study found that agreeableness is significantly negatively correlated to psychological stress response. This may be because of human interaction: individuals high in agreeableness generally have relatively better social support systems, and they will get more support in face of a stress event. Thus, they are more likely to get the resources needed to cope with stress. In addition, because individuals with high agreeableness hold a positive attitude towards humanity, they will remain relatively optimistic about results when facing stressors. This may also be one reason of their low psychological stress responses in face of stressor.

Conscientiousness refers to our control, management and regulation of our own impulses, including traits of impartiality, orderliness, self-discipline, caution and restraint. It reflects the individuals' degree of self-control and the ability to delay gratification. Individuals of high conscientiousness exhibit traits of being organized, principled and careful. The study found that conscientiousness and behavioral stress response are significantly negatively correlated. This is closely related to the stronger self-control of high conscientiousness individuals. Furthermore, the individuals high in conscientiousness have relatively high capacity to delay gratification. Compared to others, when some stressful tasks take away some leisure time, individuals high in conscientiousness are more likely to complete the task without feeling much stressful.

\subsection{Analysis of Personality Trait and Stressor on Stress Responses}

By analyzing the regression results, the study found that four stressors (work, health, family and social) and two personality traits (openness and neuroticism) are all predictors of the total stress response, wherein neuroticism has a significantly positive effect and openness has a significantly negative effect. That is to say, neuroticism can be a risk factor to stress response, while openness can be a protective factor to it. Four stressors (work, health, family and social) and two personality traits (openness and neuroticism) are all predictors of the physiological stress response, wherein neuroticism has a significantly positive effect and openness has a significantly negative effect; four stressors (work, health, family and social)and the three personality traits (neuroticism, openness and agreeableness) are all predictors of the psychological stress response, wherein neuroticism has significantly positive effect, while openness and agreeableness have significantly negative effect; three stressors (health, family, social) and three personality traits (conscientiousness, neuroticism and openness) are all predictors of the behavioral stress response, wherein neuroticism has significantly positive effect while conscientiousness and openness have significantly negative effect. It can be seen that agreeableness and conscientiousness are both protective factors to stress responses. However, when taking stressors into consideration, openness has no predictive power on stress responses.

By analyzing the relationship between personality trait and stress, we found that individual high in openness, agreeableness, conscientiousness and low in neuroticism are more likely to promptly eliminate the negative effects brought about by stressors, while the people with personality of conservation, alienation, low conscientiousness and emotional instability are more likely to exhibit psychological, physiological and behavioral problems when facing similar stressors.

\section{Conclusion}

By a comprehensive exploration of effects of stressor and personality trait on stress response, the study obtained following conclusions:

1). Four stressors (work, health, family and social) as well as two personal traits (openness and neuroticism) are predictors of total stress response, wherein neuroticism is a positive predictor while openness a negative predictor.

2). Four stressors (work, health, family and social) and two personal traits (openness and neuroticism) are predictors of physiological stress response, wherein neuroticism is a positive predictor while openness a negative predictor. 
3). Four stressors (work, health, family and social) and three personal traits (neuroticism, openness and agreeableness) are predictors of psychological stress response, wherein neuroticism is a positive predictor while openness and agreeableness are negative predictors.

4). Three stressors (health, family and social) and the three personal traits (conscientiousness, neuroticism and openness) are predictors of behavioral stress response, wherein neuroticism is a positive predictor while conscientiousness and openness are negative predictors.

\section{References}

Ahart, A.M. (2004). Determining the student behaviors contributing to teacher stress and the relationships between these behaviors and teacher characteristics. Dissertation Abstracts International Section A: Humanities and Social Sciences, 64(11-A), 39-53. http://dx.doi.org/10.1111/apps.12021

Cardon, M.S., \& Patel, P.C. (2015). Is Stress Worth it? Stress-Related Health and Wealth Trade-Offs for Entrepreneurs. Applied Psychology, 64(2), 379-420.

Chu, X.Y., Duan, T.Y., Li, Y., Han, J., \& Han, K. (2015). The Study of Relationship between Neuroticism, Stressor and Stress Response. International Journal of Economics and Finance, 7(8).

Chu, X.Y., Ma, Z.T., Li, Y., \& Han, J. (2015). Agreeableness, Extraversion, Stressor and Physiological Stress Response. International Journal of Social Science Studies, 3(4), 78-85. http://dx.doi.org/10.11114/ijsss.v3i4.857

Costa, P.T., \& McCrae, R.R. (1992). Revised NEO Personality, Inventory and NEO Five-Factor Inventory: Professional manual. Odessa, FL: Psychological Assessment Resources.

Cui, H., \& Wang, D.F. (2007). Chinese Personality and Mental Health. Advances in Psychological Science, 15(2), 234-240

Feng, D.J., Ji, L.Q., \& Yin, Z.W. (2014). Personality, perceived occupational stressor, and health-related quality of life among Chinese judges. Applied Research in Quality of Life, 9(4), 911-921. http://dx.doi.org/10.1007/s11482-013-9277-z

Grant, S., \& Langan-Fox, J. (2007). Personality and the occupational stressor-strain relationship: The role of the Big Five. Journal of Occupational Health Psychology, 12(1), 20-33. http://dx.doi.org/10.1037/1076-8998.12.1.20

Han, J., Chu, X.Y., Song, H.C., \& Li, Y. (2014). Social Capital, Socioeconomic Status and Self-efficacy. Applied Economics and Finance, 1(2), 1-10. http://dx.doi.org/10.11114/aef.v2i1.607

Kobasa, S.C., Maddi, S.R., \& Kahn, S. (1982). Hardiness and health: A prospective study. Journal of Personality and Social Psychology, 42, 168-177. http://dx.doi.org/10.1037/0022-3514.42.1.168

Krishnakumar, A., Narine, L., Soonthorndhada, A., \& Thianlai, K. (2015). Family Stressors, Home Demands and Responsibilities, Coping Resources, Social Connectedness, and Thai Older Adult Health Problems: Examining Gender Variations. Journal of Aging and Health, 27(2), 257.

Millsm, L.B., \& Huebnerm, E.S. (1998). A prospective study of personality characteristics, occupational stressors, and burnout among school psychology practitioners. Journal of School Psychology, 36(1), 103-120. http://dx.doi.org/10.1016/S0022-4405(97)00053-8

Van der Velden, P.G., Bosmans, M.W.G., Bogaerts, S., \& van Veldhoven, M.J.P.M. (2014). Social organizational stressors and post-disaster mental health disturbances: A longitudinal study. Psychiatry Research, 219(1), 177-182. http://dx.doi.org/10.1016/j.psychres.2014.05.032 